\title{
Thanks to reviewers in 2019
}

\section{Nicola Oberbeckmann-Winter ${ }^{1}$}

Published online: 21 November 2019

(C) Springer-Verlag GmbH Germany, part of Springer Nature 2019

The mission of Analytical and Bioanalytical Chemistry (ABC) is the communication of excellent research work from all fields of analytical and bioanalytical chemistry. Clearly, a scientific journal's greatest responsibility toward the scientific community is to ensure that all contributions accepted for publication are rigorously but fairly reviewed. For this reason, all manuscripts published in the journal are reviewed by expert referees. The final quality of papers accepted for publication depends to a considerable extent on the reviewers, who give valuable constructive criticism to the authors. The input and dedication of all our reviewers are highly appreciated by the editors, authors and readers of the journal.

At the end of 2019, we gratefully acknowledge the valuable support of the following scientists who have reviewed papers for the journal during the past 12 months:

Aamouche, A

Abad-Fuentes, A

Adam, $\mathrm{V}$

Adami, G

Adams, A

Adams, FC

Addepalli, B

Adıgüzel, Y

Agostini, M

Aguilar-Caballos, MdlP

Agyei, D

Ahmadi, SH

Akl, MA

Akyuz, S

Alarcón Ángeles, G

Albert, J

Albuquerque, CDL

Aldini, G

Alexandrov, T

Algar, WR

Alijanianzadeh, M
Alladio, E

Allinson, $\mathrm{G}$

Allmaier, G

Alvarez-Lorenzo, C

Amirav, A

Amjadi, M

Amoresano, A

Anderson, C E

Anderson, J L

Andersson, J T

Andrade de Lima, M

Andrisano, V

Anfossi, L

Ansell, R J

Aqel, A

Aranda, M

Araujo-Andrade, $\mathrm{C}$

Arce, L

Ariese, $\mathrm{F}$

Armenta, S

Armirotti, A
Armstrong, D

Arrua, D

Arsene, C

Astarita, G

Attygalle, A B

Avino, $\mathrm{P}$

Azimzadeh, M

Badea, G I

Badía, R

Baggiani, C

Bagheri, $\mathrm{H}$

Bai, Y

Baker, E

Baldini, F

Baluya, D

Bamba, T

Banoub, J

Bao, N

Barbas, C

Barderas, R

Nicola Oberbeckmann-Winter

nicola.oberbeckmann-winter@springer.com

1 Analytical and Bioanalytical Chemistry, Springer Verlag,

Tiergartenstrasse 17, Heidelberg 69121, Germany 
Barding, G

Barek, J

Barillaro, G

Barr, D B

Barreca, S

Barron, D

Barron, L P

Barroso, $\mathrm{M}$

Bartlett, M

Bayona, J M

Beach, D G

Beccaria, M

Beck, A

Beckmann, M

Beitollahi, H

Beklemishev, M

Beljebbar, A

Bellvert, F

Berben, G

Berezin, M

Berger, $\mathrm{S}$

Berger, TA

Berlina, A N

Bermejo Barrera, $\mathrm{P}$

Bern, M

Berti, F

Bertoncello, P

Bettmer, J

Bhardwaj, C

Biancotto, $\mathrm{G}$

Bicchi, C

Bichon, E

Bilewicz, R

Birch, B

Birolo, L P

Bischoff, R

Blanco-López, M C

Bleiholder, $\mathrm{C}$

Bocklitz, T

Bocková, M

Bodemer, W

Bodoki, E

Bogialli, S

Bohn, P

Bojko, B

Bokhart, M T

Bon, D

Bongiorno, D

Bonhommeau, S

Borchers, C H

Bordel, N

Borisov, R

Borisov, S M
Borsdorf, H

Bossi, A

Botelho, JC

Boulyga, S

Boye, S

Braissant, O

Breidbach, A

Breitkreitz, M C

Brenna, J T

Brennan, D

Brereton, R

Brett, C M A

Britz-McKibbin, $\mathrm{P}$

Broadwater, M

Brodbelt, J

Broeckling, C D

Brown, K E

Brown, S D

Brożek-Mucha, Z

Bruderer, T

Buchberger, W

Buckley, B

Bunk, D M

Bunkoed, O

Burkin, M A

Burmistrova, N A

Buszewski, B

Byrne, H J

Byzova, N A

Cabooter, D

Cacciola, F

Cagliero, $\mathrm{C}$

Calvano, C D

Camara, J E

Câmara, J S

Campbell, L

Campos-Gimenez, E

Campuzano, S

Canela-Garayoa, R

Cantafora, A D

Cao, C

Cao, $\mathrm{X}$

Capitan-Vallvey, L F

Cappa de Oliveira, L F

Capriotti, A L

Carasek, E

Cárdenas Aranzana, S

Careri, M

Carper, WR

Carrasco, S

Carrasco-Pancorbo, A

Carro, AM
Carter, C

Casale, M

Castro-Rios, R

Cataldi, T R I

Causon, $\mathrm{T}$

Ceccarelli, $\mathrm{M}$

Cela Torrijos, $\mathrm{R}$

Ceslova, L

Cestelli Guidi, M

Chailapakul, O

Chamkasem, N

Champion, J

Chan, K L A

Chang, H-T

Chankvetadze, B

Chaurand, P

Chen, A

Chen, B

Chen, C-J

Chen, J

Chen, L

Chen, S

Chen, W

Chen, Y

Chen, Z

Cheng, G

Cheng, L

Cheng, Z

Chi, Y

Chiaia-Hernandez, A C

Chiriac, $\mathrm{H}$

Cho, T J

Choi, H-K

Choi, J R

Christopoulos, $\mathrm{T}$

Chua, $\mathrm{H} \mathrm{C}$

Chuang, K-H

Chung, S W C

Cialla-May, D

Cinta Pinzaru, S

Citterio, D

Clark, K D

Clowers, B

Coelho, C

Coffinier, Y

Cologna, S M

Coltro, W

Combès, A

Concheiro-Guisan, M

Connelly, JT

Contado, C

Conte, L

Conti Gea, O 
Cooks, R G

Corbisier, $\mathrm{P}$

Cordero, C

Costa-Fernández, J M

Coy, S L

Coym, J W

Cozzolino, D

Craig, D

Cramer, B

Crimmins, B S

Cristea, C V

Crotti, S

Cubadda, F

Cui, $\mathrm{H}$

Culha, M

Cunha, S

D'Archivio, A A

D'Silva, P

Da Silva, J P

da Silva, R

Dabrio Ramos, $\mathrm{M}$

Dai, X

Dan, $X$

Dang, F

Dasgupta, P

Dastan, D

Davies, S R

Dawson, K A

Dayon, L

de la Fuente, J M

de la Torre, $\mathrm{X}$

De Loose, $\mathrm{M}$

de los Santos Álvarez, N

De Pauw, E

De Simone, S G

de Villiers, A

De Winter, J

Debode, F

Deceuninck, $Y$

Degano, I

Deiss, F

del Rio Castillo, E

del Valle Palomo Ruiz, M

Delatour, $\mathrm{V}$

Delmonte, $\mathrm{P}$

Demeke, $\mathrm{T}$

Demirev, $\mathrm{P}$

Deng, $\mathrm{C}$

Deng, $\mathrm{H}$

Deng, Q

Deo, S K

Desaire, $\mathrm{H}$
Descalzo, A B

Dickert, F L

Dillen, L

Dina, $\mathrm{N}$

Dinesh, B

Ding, L

Ding, S-N

Dixon, D

Do, T D

Dobnik, D

Dodds, J

Doetzer, R

Donato, $\mathrm{P}$

Dong, $\mathrm{C}$

Dong, F

Donnarumma, D

D'Orazio, G

Dordick, JS

Dostalek, J

Dotsikas, Y

Dou, M

Dovichi, N J

Draper, W M

$\mathrm{Du}, \mathrm{J}$

Du, P

Duan, Y

Duarte, F A

Duerkop, A

Duewer, D

Dufva, M

Dumas, $\mathrm{P}$

Dzantiev, B B

Dziadosz, $\mathrm{M}$

Easley, C J

Edwards, K A

Ehrenberg, $\mathrm{H}$

Eichhorn, K-J

El Hadri, $\mathrm{H}$

Elliott, C T

Emons, $\mathrm{H}$

Emslie, K R

Enderlein, J

Englert, D

Erden, P E

Eremin, S A

Esfandiari, L

Esteve-Romero, J

Esteves da Silva, J C G

Evans-Nguyen, K M

Ewing, A

Fabbris, L
Fakhrullin, R F U

Famiglini, G

Fan, $\mathrm{H}$

Fan, Y

Fanali, C

Fang, N

Fang, Q

Farhane, Z

Faria, $M$

Fattorini, $\mathrm{P}$

Favretto, D

Fechete, R

Fechner, $\mathrm{P}$

Fedorova, M

Feldmann, J

Feng, W

Feng, Y-Q

Fernanda Silva, $\mathrm{M}$

Fernandez, B

Fernandez, F M

Fernandez, J A

Fernandez Abedul, M T

Fernandez-Lafuente, R

Fernandez-Lima, F

Fernández-Sánchez, J F

Ferreira, C R

Fetzer, J C

Fiehn, O

Filik, $\mathrm{H}$

Fillet, M

Flora, J W

Flores, E M d M

Fontanals, N

Fornstedt, $\mathrm{T}$

Forsythe, J G

Franco, R

Fratila, R M

$\mathrm{Fu}, \mathrm{Y}$

Gallardo, E

Gallello, G

Galligan, T M

Gamella, M

Gan, N

Gao, B

Gao, P

Gao, Q

Gao, X

Garcia-Villalba, R

Garnier, N

Garrard, K

Garrido, M

Garvey, J 
Gaspar, E M M S M

Gautam, N

Ge, J

Gee, $\mathrm{P}$ T

Gee, S J

Genc, $\mathrm{R}$

Gerber, C

Gescheidt, G

Ghosh, A

Giannetti, A

Gibson-Daw, G

Giera, M

Gil, A M

Gilar, M

Gilchrist, M L

Gionfriddo, E

Giovannoli, C

Giovanoulis, G

Giraudeau, P

Gleisner, $\mathrm{H}$

Godin, S

Göen, $\mathrm{T}$

Goenaga-Infante, $\mathrm{H}$

Goicoechea, H C

Gong, $\mathrm{M}$

Gonzalez-Iglesias, $\mathrm{H}$

González-Ruiz, V

González-Sálamo, J

Goodpaster, J V

Gordon, K

Gorris, H H

Goryacheva, I Y

Gottwald, S

Graham, S

Granato, D

Grant, E

Grasso, G

Grechnikov, A

Grigoryev, A

Grimalt, J

Grinias, J P

Gronwald, W

Gross, J H

$\mathrm{Gu}, \mathrm{J}$

$\mathrm{Gu}, \mathrm{Y}$

$\mathrm{Gu}, \mathrm{Z}$

Guan, F

Guan, Y

Guchelaar, H-J

Guevara, E

Guijt, R

Guillarme, D

Gundlach-Graham, A
Guo, J

Guo, L

Guo, M

Guo, Y

Guo, Z

Guttena, V

Haag, M

Hall, A J

Halvorsen, T G

Han, D

Han, Y

Hanai, T

Hare, D J

Harrington, P d B

Harynuk, J J

Hasegawa, $\mathrm{H}$

Hauser, P C

Hausler, $\mathrm{P}$

Hawkridge, A

Hayen, $\mathrm{H}$

Hayes, M

$\mathrm{He}, \mathrm{G}$

$\mathrm{He}, \mathrm{R}$

He, $\mathrm{X}$

He, Y

Heaney, L M

Heberger, K

Hegemann, A D

Heijs, B

Heiss, C

Heltai, G

Hemmer, E

Henrion, A

Henry, C

Hepel, M

Hercules, D M

Hernandez, F

Hernández Borges, J

Hernandez-Cordoba, M

Herr, A

Herrero, M

Herzsprung, $\mathrm{P}$

Hianik, T

Hilder, E

Hill, S

Himmelsbach, M

Hitzmann, B

Ho, K-S

$\mathrm{Ho}, \mathrm{SSH}$

Hoijtink-Huijbrechts, A M L

Holčapek, M

Holland, L A
Hong, S

Hongsibsong, S

Hopfgartner, G

Horstkotte, B

Hosseini, M

Hsu, F F

$\mathrm{Hu}, \mathrm{B}$

$\mathrm{Hu}, \mathrm{Q}$

$\mathrm{Hu}, \mathrm{S}$

$\mathrm{Hu}, \mathrm{W}$

$\mathrm{Hu}, \mathrm{Y}$

Huang, D

Huang, F

Huang, G

Huang, L

Huang, Q

Huber, C G

Hughes, C C

Huhn, C

Hummon, A B

Huo, F

Hupp, A M

Hurth, C

Hutterer, J

Hutzler, C

Huynh, K

Ibañez, E

Ifa, D R

Igne, B

Ikegami, T

Inagaki, K

Indrasekara, $\mathrm{S}$

Infante, C

Iqbal, N

Itoh, $\mathrm{T}$

Ivleva, N P

Ivory, $\mathrm{C}$

Jackson, G P

Jackson, S N

Jain, $P$

Janfelt, C

Jang, H W

Jankowski, K J

Jaoui, M

Jebakumar Immanuel Edision, T

Jehlicka, J

Jia, L

Jia, S

Jiang, H

Jiang, J

Jiang, $\mathrm{X}$ 
Jin, L

Jin, R

Jin, $\mathrm{S}$

Jin, W

Jin, Y

John, H

Johnson, R

Jones, J J

Jones, S H

Josephs, R D

Joshi, A

Joshi, M D

Ju, Y

Jun, B-H

Jung, J

Jungnickel, H

Jurado Sánchez, B

Juskowiak, B

Kadimisetty, K

Kafouris, D

Kailasa, S K

Kaljurand, M

Kalogianni, D P

Kaltashov, I A

Kampf, C J

Kang, J

Kang, T

Kanický, V

Kapusta, P

Kara, P

Karioti, A

Kasprzyk-Hordern, B

Kavakiotis, I

Kawasaki, H

$\mathrm{Ke}, \mathrm{R}$

Ke, S

Kennedy, R T

Kerman, K

Kerscher, R

Kessler, R W

Kiefer, J

Kim, B

Kim, J

Kim, S

Kind, T

Kintz, $\mathrm{P}$

Kirsanov, D O

Kitagawa, S

Kjelland, M E

Klampfl, C W

Kneipp, J

Knepper, T P
Knopp, D

Kobrak, M N

Koch, H M

Koch, M

Kochana, J

Koenig, A

Köfeler, H

Kole, P L

Konarski, P

König, S

Korfmacher, W

Kos, G

Kosman, J

Koziel, J A

Kraemer, T

Kramer, K

Kramer, U

Kranz, C

Krauss, M

Kricka, L

Kroflic, A

Krska, R

Krupadam, R J

Krylov, S N

Kubatova, A

Kuesters, M

Kuhn, A

Kuklenyik, Z

Kumar, J R

Kumke, M U

Kunz, W

Kuo, C-H

Kuo, T-R

Kylin, $\mathrm{H}$

Laborda, F

Lacorte, S

Laemmerhofer, $\mathrm{M}$

Laganà, A

Lagarde, F

Lamari, F

Lammertyn, J

Lamminmäki, U

Lanças, F M

Lang, C

Lankova, D

Lao, W

Lapizco-Encinas, B H

Lascola, R

Lavine, B K

Le, XC

Leal, W

Leblanc, R M
Lebrilla, C

Lederer, A

Lee, H K

Lee, $\mathrm{H} \mathrm{S}$

Lee, $\mathrm{Y}$

Lee, Y-J

Legland, D

Lehmann, W D

Lehnert, R

Lehotay, S J

Lei, J

Lei, Y

Leitner, A

Lenz, D

Leopold, K

Leopold, N

Lerner, D A

Lesur, A

Lhotská, I

Li, B

Li, D-W

Li, F

Li, G

Li, H

Li, J

Li, J-F

Li, L

Li, P

Li, W-Y

Li, X

Li, Y

Li, Z

Lieberman, M

Lieberzeit, P A

Ligor, T

Lim, K M

Limbeck, A

Lin, C-H

Lin, Z

Linden, R

Linhardt, R J

Linsinger, T P J

Lintelmann, J

Lipka, E

Lisdat, F

Lisec, J

List, $\mathrm{M}$

Litescu, S C

Litsardakis, G

Liu, F

Liu, H

Liu, J

Liu, Q 
Liu, R

Liu, Y

Liu, Y-Q

Liu, Z

Lobo-Castañón, M J

Locatelli, M

Lockett, M R

Lockridge, $\mathrm{O}$

Lofblom, J

Long, $\mathrm{S}$

Longuespée, $\mathrm{R}$

Loo, JA

Lopez Guerrero, M M

López-Alarcón, C

Loppnow, G R

Lorenzo, $\mathrm{R}$

Lorenzo Abad, M E

Lough, J

Loziuk, P

$\mathrm{Lu}, \mathrm{C}$

$\mathrm{Lu}, \mathrm{F}$

Lu, $\mathrm{H}$

$\mathrm{Lu}, \mathrm{J}$

$\mathrm{Lu}, \mathrm{X}$

$\mathrm{Lu}, \mathrm{Y}$

Lucena Rodríguez, $\mathrm{R}$

Luch, A

Lucklum, R

Luque-Garcia, J L

Lv, Y

Ma, J

Ma, L

Ma, M

Maas, A

Madariaga, J M

Magni, S

Mahmud, I

Maia, M

Maitre, $\mathrm{P}$

Malenovic, A

Malhotra, B D

Malinovskiy, D N

Malitesta, C

Mallard, G

Manfredi, M

Mani, V

Maquieira, Á

Marć, M

Marce, R M

Marcelli, A

Marchetti-Deschmann, M

Marcus, R K
Marczylo, T

Marina, M L

Marini, F

Marken, $F$

Markin, A V

Marshall, A G

Marshall, C

Marshall, J

Martin, F L

Martín-Esteban, A

Martinez Carballo, E

Maspoch, S

Massolini, G

Mathwig, K

Mattarozzi, M

May, JC

Mayboroda, OA

Mayer, $\mathrm{M}$

McComb, M

McCord, B R

McDonnell, L

McGown, L

McLean, J A

Meade, A D

Meagher, R J

Melanson, J E

Men, D

Mendieta-Wejebe, J E

Mercier, F

Mercl, F

Mercolini, L

Merk, V

Meyer, M R

Miao, $\mathrm{P}$

Micheli, L

Michelini, E

Millet, $\mathrm{M}$

Minor, E

Miralles Buraglia, B

Miranda Castro, R

Mirkin, C A

Mishra, Y K

Misra, B B

Mitchell, J

Mitić, Ž J

Mittermaier, A

Mitulovic, G

Miyaguchi, $\mathrm{H}$

Mnatsakanyan, $\mathrm{M}$

Möder, M

Modugno, F

Mohammadi, M

Molinié, R
Moltó, J C

Monaci, L

Montoro Bustos, A R

Moon, $\mathrm{M} \mathrm{H}$

Moore, D S

Morais, S

Moreno Bondi, M C

Morlock, G E

Moscone, D

Moyano, E

Mudalige, $\mathrm{T}$

Mukherjee, A

Muller, L

Müller-Platz, C

Murray, K K

$\mathrm{Na}, \mathrm{N}$

Namiesnik, J

Näreoja, T

Natividad, M T

Navarro, F

Nazar, M F

Nazari, M

Neal, S L

Nectoux, J

Neu, T R

Neugebauer, U

Nic Daeid, N

Niccolucci, F

Nichols, D S

Nie, L

Nie, Z

Nielen, $M$

Niessner, R

Nihonyanagi, S

Nikolic, D

Nilsson, L

Nitride, C

Nobrega, J A

Nojima, Y

Nöll, G

Nordborg, A

Nowak, S

Nugen, S R

Numata, M

Nuzillard, J-M

Oberthuer, D

O'Connor, G

O'Connor, P

Oelkrug, D

Ogra, Y

Oleschuk, R 
Oliveira, H M

Oprea, A

Orinakova, R

Osipov, A P

O'Sullivan, C K

Ouyang, G

Ouyang, Z

Owens, R

Ozalp, V C

Ozkan-Ariksoysal, D

Pabst, M

Paczkowski, S

Padula, M P

Pagel, K

Palacio Lozano, D C

Palchetti, I

Pallaoro, A

Palleschi, V

Pallua, J

Palma, $P$

Palmer, C

Palzer, S

Pan, S-D

Panchagnula, V

Panderi, I E

Panikkanvalappil, S

Panne, U

Park, J P

Pascale, M

Paseiro Losada, P

Pasti, L

Patti, A

Paunesku, T

Pechprasarn, S

Pedrero, M

Pei, $\mathrm{H}$

Peltomaa, R

Peluso, $\mathrm{P}$

Pentieva, K

Pereiro, R

Pérez-Parada, A

Perry, M

Perullini, $\mathrm{M}$

Peters, FT

Petersen, E

Petr, J

Pfeiffer, C

Phillips, M M

Phinney, K W

Pichon, V

Picó, Y

Pietrodangelo, A
Pingarrón, J M

Pino, V

Pischetsrieder, $\mathrm{M}$

Pisonero, J

Pitkänen, L

Pitteri, S

Plasek, J

Plavec, J

Pleadin, J

Pleil, J

Plenis, A

Pohl, N

Polesello, S

Polewski, K

Popov, A M

Popov, I A

Popp, J

Poppi, R J

Posthuma-Trumpie, GA

Potter, O

Pozo, O J

Prasse, C

Primpke, $\mathrm{S}$

Prohaska, T

Proll, G

Prosen, $\mathrm{H}$

Provenzano, M

Psillakis, E

Purcaro, G

Purves, R W

Pyo, J S

Qi, H

Qian, J

Qian, W-J

Qiao, J

Qiao, X

Qiqin, W

Qiu, B

Qiu, J

$\mathrm{Qu}, \mathrm{F}$

$\mathrm{Qu}, \mathrm{G}$

Qu, L-L

Que, L

Quintas, G

Quinto, M

Quirino, J P

Rabin, I

Radke, W

Raghavan, S

Ragno, G

Rajabi, H R
Rajabi, M

Ramanavicius, A

Ramos, L

Ramos, R M

Rampler, E

Rathore, A S

Ray, T

Regal, $\mathrm{P}$

Reiner, J L

Ren, J

Ren, $\mathrm{K}$

Ren, $\mathrm{Y}$

Resano, M

Reschiglian, $\mathrm{P}$

Restolho, J

Reyns, T

Reyzer, M L

Ricci, F

Richards Kortum, R

Richter, M

Rigano, $\mathrm{F}$

Rios-Castro, A

Robb, P

Robbat, A

Rödiger, $\mathrm{S}$

Rodriguez-Gonzalez, $\mathrm{P}$

Rohnke, M

Romero-Gonzalez, R

Rong, G

Rösch, P

Rosen, E

Rosero, $\mathrm{M}$

Roshal, A

Rosi, F

Roupioz, Y

Roy, D

Roy-Lachapelle, A

Ruan, C

Rubim, J C

Rubiolo, $\mathrm{P}$

Ruckebusch, C

Rüger, C P

Ruhaak, L R

Rurack, K

Saba, A

Sabbatini, L

Sahoo, H R

Sahoo, S

Sahore, V

Saint-Marcoux, F

Saito, $T$

Saito, Y 
Sajid, M

Salimi, A

Salinas-Castillo, A

Salminen, J-P

Salvador, J-P

Salzer, R

Samal, S K

Sanchez, L

Sancho, J V

Sander, L C

Sandra, K

Sandra, P

Sandrin, T

Santer, J

Saraji, M

Šatínský, D

Sauve, S

Sayyadi, N

Scarano, S

Schäferling, M

Schambeau, L

Schantz, M

Schebb, N H

Scheeline, A

Scheper, T

Schiel, J

Schierbaum, K

Schmid, R

Schmidt, T C

Schmitz, O J

Schneider, R J

Schug, K A

Schunck, W H

Schürch, S

Schuster, M

Schweiggert, R M

Sciarrone, D

Seah, M P

Seeley, J

Segundo, M A

Seidel, M

Seidi, S

Seitz, W

Seo, J H

Sha, J-J

Shallan, A

Shalliker, A

Shang, L

Shangguan, D

Shao, S

Shen, A-G

Shen, $F$

Sheta, S M
Shi, F

Shi, W

Shi, X

Shiddiky, M J A

Shieh, D-B

Shih, S

Shimma, S

Shoji, M

Shuford, C M

Siluk, D

Silva, M F

Simat, T

Simó-Alfonso, E F

Singh, D R

Singh, N

Siripinyanond, A

Skladal, P

Skopp, G

Smichowski, P N

Smith, E

Smith, R M

Snow, N

Snyder, N

Sobek, J

Sojic, N

Sojo, L E

Solich, P

Soltwisch, J

Song, Y

Soylak, M

Sparvero, L J

Spence, D M

Sperling, M

Spooner, N

Spoto, G

Sprakel, L

Springer, T

Stalikas, C D

Stanković, D M

Stärk, H-J

Steiner, G

Steinwand, M

Stella, R

Stepien, E

Stewart, A

Stewart, C F

Stobiecka, M

Stove, C P

Stübiger, $\mathrm{G}$

Studzinska, S

Sturtevant, D

Stürup, S

$\mathrm{Su}, \mathrm{B}$
Su, L

Su, X

Su, Z

Sugimoto, I

Sui, Z

Sun, C

Sun, L-N

Sun, $M$

Sun, S

Supekar, N T

Suter, MJ-F

Sutherland, K

Svec, F

Švore, L'

Szostak, R

Szterk, A

Szumski, M

Szunerits, $S$

Szymborski, T

Taghdisi, S M

Takarada, T

Takatsu, A

Tamer, U

Tan, J

Tan, Y

Tang, D

Tang, L

Tanner, J

Tao, S

Taraji, M

Taverniers, I

Taylor, L

Taylor, S L

Teo, A

Thevis, $\mathrm{M}$

Thompson, $\mathrm{P}$

Thompson, $\mathrm{T}$

Tian, F

Tian, Z

Tirler, W

Tombelli, S

Tong, C

Torta, F

Touboul, D

Tranchida, P Q

Trejos, $\mathrm{T}$

Tripathy, U

Tripodi, V

Trovato, E

Trtic-Petrovic, T M

Tsargorodska, A

Tsim, K W K 
Tsugawa, $\mathrm{H}$

Tu, A

Tuma, $\mathrm{P}$

Turner, $\mathrm{C}$

\section{Ugarova, $\mathrm{N} \mathrm{N}$}

Ulmer, C Z

Ulrich, $\mathrm{S}$

Ul'yanovskii, N V

Unnikrishnan, V K

Urraca, J L

Utczas, M

Vacca, G

Vaculovic, T

Vadillo, J M

van den Berg, F

van Drooge, B L

Van Orden, A

Vanhaecke, L

Vanna, R

Vashist, S K

Veenaas, C

Velazquez-Campoy, A

Venkatramani, C J

Vermathen, M

Verona, E

Vetter, W

Vianello, F

Villalonga, R

Villani, C

Vogel, M

Vogl, J

Voitechovic, E

Volk, D E

Vuckovic, D

Vyskocil, V

Wagner, J R

Wagner, M

Walch, A

Walker, M

Wan, D

Wang, A-J

Wang, F

Wang, $\mathrm{H}$

Wang, H-B

Wang, H-S

Wang, H-Y J

Wang, J

Wang, L

Wang, M

Wang, Q
Wang, S

Wang, W

Wang, $\mathrm{X}$

Wang, Y

Wang, Y-Q

Wang, Z

Warren, J

Wasik, A

Waterhouse, A L

Webb, B A

Weber, G

Weber, S G

Wegscheider, W

Wei, G

Wei, H

Wei, Q

Weidner, S

Weinberger, $\mathrm{K}$

Weissberg, A

Weller, M G

Wen, E

Weng, S

Wentao, X

Wernisch, S

West, C

Westwood, S W

White, I

Wickremsinhe, E R

Wieczorek, P P

Wielgomas, B

Wilhelm, S

Wilkins, J A

Wille, S M R

Williams, P J

Willis, $\mathrm{P}$

Willner, I

Wilson, S R H

Wiltsche, $\mathrm{H}$

Winterhalter, $\mathrm{P}$

Witt, M

Wolfbeis, O S

Workman, W E

Wrobel, K

$\mathrm{Wu}, \mathrm{H}$

$\mathrm{Wu}, \mathrm{J}$

Wu, J-Y

Wu, R

Wu, Z-Y

Wuethrich, A

Xia, F

Xia, X

Xia, Y
Xiao, H

Xiao, L

Xiao, Y

Xiaobo, Z

Xie, J

Xie, Q

Xie, Y

$\mathrm{Xu}, \mathrm{D}$

$\mathrm{Xu}, \mathrm{F}$

$\mathrm{Xu}, \mathrm{G}$

$\mathrm{Xu}, \mathrm{L}$

$\mathrm{Xu}, \mathrm{T}$

$\mathrm{Xu}, \mathrm{W}$

$\mathrm{Xu}, \mathrm{X}$

$\mathrm{Xu}, \mathrm{Z}$

Xu, Z-R

Xuan, X

Xue, M

Xue, R

Yagati, A

Yamini, Y

Yan, B

Yan, $\mathrm{X}$

Yanan, D

Yañez-Sedeño Orive, $\mathrm{P}$

Yang, F-Q

Yang, J

Yang, L

Yang, M

Yang, W

Yang, Y

Yang, $\mathrm{X}$

Yao, W

Yao, Y

Ye, D

Ye, F

Ye, Y

Yeow, E

Yi, C

Yi, F

Yi, L

Yim, Y-H

Yin, P-G

Yin, X-B

Yoo, $\mathrm{HH}$

You, M

Yu, C-J

$\mathrm{Yu}, \mathrm{F}$

Yu, H

Yu, J

Yu, L L

Yu, L-P 
$\mathrm{Yu}, \mathrm{Y}$

Yu, Y-L

Yuan, B

Yuan, G

Yuan, L

Yuan, M

Yusà, V

Zablackis, E

Zahavy, E

Zaia, J

Zare, R

Zarrine-Afsar, A

Zelada-Guillen, G

Zeleny, R

Zeng, A

Zeng, G

Zeng, $\mathrm{H}$

Zenkia, O V

Zhang, B
Zhang, D

Zhang, F

Zhang, $\mathrm{H}$

Zhang, J

Zhang, K

Zhang, L

Zhang, Q

Zhang, Q-L

Zhang, S

Zhang, W

Zhang, $X$

Zhang, $\mathrm{Y}$

Zhao, B

Zhao, H

Zhao, H Z

Zhao, L

Zhao, Q

Zhao, S

Zhao, W

Zhao, X
Zhao, Y-Y

Zhao, Z

Zheng, C

Zheng, J

Zheng, W

Zhong, W

Zhou, L

Zhou, Q-X

Zhou, T

Zhou, X

Zhu, G

Zhu, J

Zhu, J-J

Zhu, Y

Zhu, Z

Zhu, Z-J

Zoccali, M

Zonja, B

Zweigenbaum, J

Zwiener, C 